Pacific Journal of Mathematics

REALIZING PARTIAL ORDERINGS BY CLASSES OF
CO-SIMPLE SETS

Jere Brian Rem ute 


\title{
REALIZING PARTIAL ORDERINGS BY CLASSES OF CO-SIMPLE SETS
}

\author{
J. B. REMMEL
}

We show that we can embed any countable partial ordering into a class of co-r.e. bi-dense subsets of the rationals, each subset of a fixed nonzero r.e. Turing degree, under an order induced by recursive similarity transformations. Also, we show that we can embed any countable partial ordering into the co-simple isols under either the order induced by addition of isols or the order induced by recursive injections.

o. Introduction. Let $C$ denote the continuum, $Q$ denote the rationals, and $N$ denote the natural numbers. We let $c$ denote the cardinality of $C$ and $\aleph_{0}$ denote the cardinality of $N$. Given two linear orderings $H$ and $G$, we say (i) $H$ is embeddable in $G, H<G$, if there is an order preserving map from $H$ into $G$ and (ii) $H$ is similar to $G$ if there is an order preserving map from $H$ onto $G$. $H$ is said to be bi-dense in $G$ if $H \subseteq G$ and both $H$ and $G-H$ are dense in $G$.

Let $\pi$ be an effective one-one correspondence between $Q$ and the natural numbers. We shall consider $\pi$ to be an effective Gödel numbering and thus we will identify an element or subset of $Q$ with its image under $\pi$. We let $\leqq$ or $<$ refer to the usual ordering on $N$ and $(\Leftrightarrow)$ or $(\diamond)$ refer to the usual ordering on $Q$. Given $\alpha, \beta \subseteq Q$, we say $\alpha$ is recursively embeddable in $\beta, \alpha \prec_{c} \beta$, if there is a partial recursive function $\rho$ such that $\alpha \cong \delta \varphi$, the domain of $\varphi$, and the restriction of $\phi$ to $\alpha, \varphi \uparrow \alpha$, is an order preserving map from $\alpha$ into $\beta$.

In [5], Hay, Manaster, and Rosenstein show that complements of recursively enumerable bi-dense subsets of $Q$ of any fixed nonzero r.e. degree under $<_{c}$ bear a strong resemblance to bi-dense subsets of $C$ of cardinality $c$ under $\prec$. The main result of this paper answers a question raised by Laver. Based on the results of [5], Laver asked whether or not the following theorem is true.

THEOREM A. Let $\beta$ be any recursively enumerable set which is not recursive and let $P$ be any countable partial ordering. Then there is a collection of co-recursively enumerable bi-dense subsets of $Q$, each Turing equivalent to $\beta$, such that, under $<_{c}$, this collection is order isomorphic to $P$.

(A set $A \subseteq N$ is co-recursively enumerable if $N-A$ is recursively enumerable.) In $\S 2$ of this paper, we prove Theorem A using methods that Sack's [8] developed to prove that any countable partial ordering 
can be embedded in the r.e. Turing degrees under the order induced by Turing reducibility. Theorem A extends Theorems 7 and 8 of [5], where Hay, Manaster, and Rosenstein proved the analogues of Theorem $\mathrm{A}$ if the countable partial ordering $P$ in the statement of Theorem A is replaced either by any countable linear ordering or by any finite partial ordering.

The proof of Theorem A will also give a result on the class of co-r.e. isols which have been studied by Hay [3], [4], Ellentuck [2], and others. We will show that one can embed any countable partial ordering $P$ into the class of co-simple isols under either the order induced by addition of isols (due to Ellentuck [2]) or the order induced by recursive injections. (See $\S 1$ for the definitions of the co-simple isols and the two orderings.)

1. Preliminaries. Given $B \subseteq N$, we write $\bar{B}$ for the complement of $B$ in $N$. We write $A \leqq_{T} B$ if $A$ is Turing reducible to $B$ and $A \equiv_{T} B$ if $A \leqq_{T} B$ and $B \leqq_{T} A$. Let $\varphi_{0}, \varphi_{1}, \cdots$ be an effective list of all partial recursive functions where $\mathcal{\rho}_{n}$ is the function computed by the $n$th Turing machine. We write $\varphi_{n}^{s}(x) \downarrow$ if the $n$th Turing machine started on $x$ gives an output in $s$ or less steps. We let $I_{0}, I_{1}, \cdots$ be an effective list of all intervals of $Q$ of the form $[p, q]=$ $\{x \in Q \mid p \triangleq X \lesssim q\}$ for $p, q \in Q$.

Given a partial ordering $P$, we say $P$ is an $\aleph_{0}$-universal partial ordering if any countable partial ordering can be embedded in $P$, that is, if $S<P$ for all countable partial orderings $S$. The rest of this section will be devoted to defining three partial orderings. The fact that each of the three partial orderings is $\boldsymbol{\aleph}_{0}$-universal will follow easily from the main construction of $\S 2$.

Given $\alpha, \beta \leqq Q$, we define $\alpha \sim_{c} \beta$ iff $\alpha \prec_{c} \beta$ and $\beta \prec_{c} \alpha$. It is clear that $\sim_{0}$ is an equivalence relation. Let $\boldsymbol{a}$ be any nonzero r.e. Turing degree. We let $B(\boldsymbol{a}, Q)=\{\alpha: \alpha$ is a co-r.e. bi-dense subset of $Q$ of degree $\boldsymbol{a}\}$ and $\bar{B}(\boldsymbol{a}, Q)=B(\boldsymbol{a}, Q) / \sim_{c}$. Given equivalence classes, $[\alpha],[\beta] \in \bar{B}(\boldsymbol{a}, Q)$, we define $[\alpha] \leqq_{c}[\beta]$ iff there exists $\alpha \in[\alpha]$ and $\beta \in[\beta]$ such that $\alpha \prec_{c} \beta$. It is easy to check that $\leqq_{c}$ is a well defined partial order on $\bar{B}(\boldsymbol{a}, Q)$. Thus, Theorem $\mathrm{A}$ is equivalent to saying that $\left\langle\bar{B}(\boldsymbol{a}, Q), \leqq_{c}\right\rangle$ is an $\boldsymbol{\aleph}_{0}$-universal partial ordering for any nonzero r.e. degree $a$.

Given $\alpha, \beta \subseteq N$, we say $\alpha$ is recursively equivalent to $\beta$ if there is a $1-1$ partial recursive function $p$ such that $\alpha \cong \delta p$ and $p \uparrow \alpha$ maps $\alpha$ onto $\beta$. The recursive equivalence type or RET of $\alpha$, denoted by $\langle\alpha\rangle$, is the class of all $\beta$ recursively equivalent to $\alpha$. A set $\alpha \subseteq N$ is immune if $\alpha$ is infinite and $\alpha$ has no infinite r.e. subset. A r.e. set $\beta \cong N$ is simple if $\bar{\beta}$ is immune. A set $\alpha \cong N$ is isolated if $\alpha$ is either finite or immune. The RETs of isolated sets are called 
isols and their collection is denoted by $\Lambda$. The elements of $\Lambda$ can be considered as an "effective" analogue of the Dedekind finite cardinals and have been extensively studied by Dekker, Manaster, Myhill, Nerode, and others. Isols $\langle\alpha\rangle$ of sets $\alpha$ such that $\alpha$ is co-r.e. are called co-simple isols and their collection is denoted by $\Lambda_{z}$. We shall define two distinct partial orders on $\Lambda_{z}$. Addition of RETs is defined by $\langle\alpha\rangle+\langle\beta\rangle=\langle\{2 x \mid x \in \alpha\} \cup\{2 x+1 \mid x \in \beta\}\rangle$. The partial ordering $\leqq_{i}$ is defined on the RETs by $A \leqq_{i} B$ iff $\exists C(A+C=B)$. Given sets $\alpha, \beta \subseteq N$, we define $\alpha \preccurlyeq_{i} \beta$ iff $\alpha \subseteq \beta$ and there are disjoint r.e. sets $W_{1}$ and $W_{2}$ such that $W_{1} \cap \beta=\alpha$ and $W_{2} \cap \beta=\beta-\alpha$. It is proved in [1], that for RETs $\langle\alpha\rangle$ and $\langle\beta\rangle,\langle\alpha\rangle \leqq_{i}\langle\beta\rangle$ iff there exists $\alpha^{\prime} \in\langle\alpha\rangle$ and $\beta^{\prime} \in\langle\beta\rangle$ such that $\alpha^{\prime} \prec_{i} \beta^{\prime}$. Given sets $\alpha, \beta \subseteq N$, we define $\alpha \prec_{e} \beta$ iff there is a partial recursive function $p$ such that $\alpha \subseteq \delta p$ and $p\lceil\alpha$ is a $1-1$ map from $\alpha$ into $\beta$. Given RETs $\langle\alpha\rangle$ and $\langle\beta\rangle$, we define $\langle\alpha\rangle \leqq_{e}\langle\beta\rangle$ iff there exists $\alpha^{\prime} \in\langle\alpha\rangle$ and $\beta^{\prime} \in\langle\beta\rangle$ such that $\alpha \prec_{e} \beta$. It is easy to check that $\leqq_{e}$ is a well defined partial order on the class of RETs.

In $\S 2$, we shall prove that $\left\langle\bar{B}(\boldsymbol{a}, Q), \leqq_{c}\right\rangle,\left\langle\Lambda_{z}, \leqq_{i}\right\rangle$, and $\left\langle\Lambda_{z}, \leqq_{e}\right\rangle$ are all $\aleph_{0}$-universal partial orderings. We shall discuss the differences between $\nvdash_{c}, \gamma_{i}$, and $\gamma_{e}$ on the class of co-r.e. sets and the differences between $\leqq_{i}$ and $\leqq_{e}$ on $\Lambda_{z}$ in $\S 3$.

2. The main construction. In [5], Hay, Manaster, and Rosenstein constructed a set $\alpha \subseteq Q$ with the following property.

$(\mathscr{P})$ If $\varphi$ is a partial recursive function such that $\alpha \cong \delta \varphi$ and $\varphi \nmid \alpha$ is a $1-1$ map from $\alpha$ into $\alpha$, then $\{\alpha \in \alpha \mid \varphi(\alpha) \neq \alpha\}$ is finite. If $\alpha$ has property $\mathscr{P}$ then $\alpha$ is isolated. For if $\alpha$ contains an infinite r.e. set, then $\alpha$ contains an infinite recursive set $R=$ $\left\{a_{0}<a_{1}<a_{2}<\cdots\right\}$. Let $\phi$ be the recursive function defined by

$$
\varphi(x)= \begin{cases}a_{i+1} & \text { if } x=a_{i} \text { and } i \text { is even } \\ a_{i-1} & \text { if } x=a_{i} \text { and } i \text { is odd } \\ x & \text { otherwise }\end{cases}
$$

$\varphi\lceil\alpha$ thus would be a $1-1$ map from $\alpha$ into $\alpha$ such that $R=$ $\{a \in \alpha \mid a \neq \varphi(a)\}$ contradicting property $\mathscr{P}$. If $\alpha$ is isolated, then $\alpha$ has the property that for no proper subset $\beta$ of $\alpha$ is $\alpha \gamma_{c} \beta$. For if $\beta \subset \alpha$ and $\alpha \prec_{c} \beta$, then let $\phi$ be the partial recursive function such that $\alpha \subseteq \delta \rho$ and $\varphi \uparrow \alpha$ is an order isomorphism from $\alpha$ into $\beta$. Let $x \in \alpha-\beta$. Thus either $x \ominus \phi(x)$ or $\phi(x) \ominus x$. If $x \ominus \phi(x)$, then $\{x \ominus$ $\varphi(x) \ominus \varphi(\varphi(x)) \otimes \varphi(\varphi(\varphi(x))) \otimes \cdots\}$ is an infinite r.e. subset of $\alpha$ and if $\varphi(x) \ominus x$, then $\{x \otimes \varphi(x) \oslash \varphi(\varphi(x)) \oslash \cdots\}$ is an infinite r.e. subset of $\alpha$ contradicting the fact that $\alpha$ is isolated. All sets $\alpha$ we construct in this section will have property $\mathscr{P}$ so that we will always have $\langle\alpha\rangle \in \Lambda$. 
The proof that $\left\langle\bar{B}(\boldsymbol{a}, Q), \leqq_{c}\right\rangle,\left\langle\Lambda_{z}, \leqq_{i}\right\rangle$, and $\left\langle\Lambda_{z}, \leqq_{e}\right\rangle$ are $\aleph_{0^{-}}$ universal ordering will proceed in two steps in the same manner as Sack's proof [8] of the fact that the r.e. degrees under Turing reducibility is an $\boldsymbol{\aleph}_{0}$-universal partial ordering. The first step is to construct an infinite sequence of 'incomparable' elements.

THEOREM 1. Let $\beta$ be a nonrecursive r.e. set. There is a recursive sequence of co-r.e. subsets of $Q, \alpha_{0}, \alpha_{0}, \cdots$, such that

(a) For each $i, \alpha_{i}$ is bi-dense in $Q$,

(b) For each recursive set $R \subseteq N, \bigcup_{i \in R} \alpha_{i}$ has property $\mathscr{P}$,

(c) For each $i, \alpha_{i} \cap \bigcup_{i \neq j} \alpha_{j}=\varnothing$ and moreover $\alpha_{i} \nless_{\hat{e}} \bigcup_{i \neq j} \alpha_{j}$, and

(d) For each $i, \alpha_{i} \equiv_{T} \beta$.

Proof. Let $f$ be a $1-1$ recursive function whose range is $\beta$ and let $\beta^{s}=\{y \mid \exists x(x \leqq s \& f(x)=y)\}$. Let $k: N \times N \rightarrow N$ be a $1-1$, onto, recursive function. Let $r$ and $c$ be recursive functions such that $k(i, j)=n$ iff $c(n)=i$ and $r(n)=j$. Moreover, we assume $k$ is chosen so that for each $i, N_{i}=\{y \mid \exists x(k(i, x)=y)\}$ is a bi-dense recursive subset of $Q$. We shall give a procedure to enumerate a r.e. set $A$ in stages such that if $\alpha_{i}=\bar{A} \cap N_{i}$, then $\alpha_{0}, \alpha_{1}, \cdots$ is the recursive sequence of sets required by the theorem. Each $\alpha_{i}$ is cor.e. since $\bar{\alpha}_{i}=\left(A \cap N_{i}\right) \cup \bigcup_{i \neq j} N_{j}$ and clearly the sets $\alpha_{0}, \alpha_{1}, \cdots$ are pairwise disjoint.

A convenient picture for the construction of $A$ will be to imagine an infinite sequence of infinite columns of windows

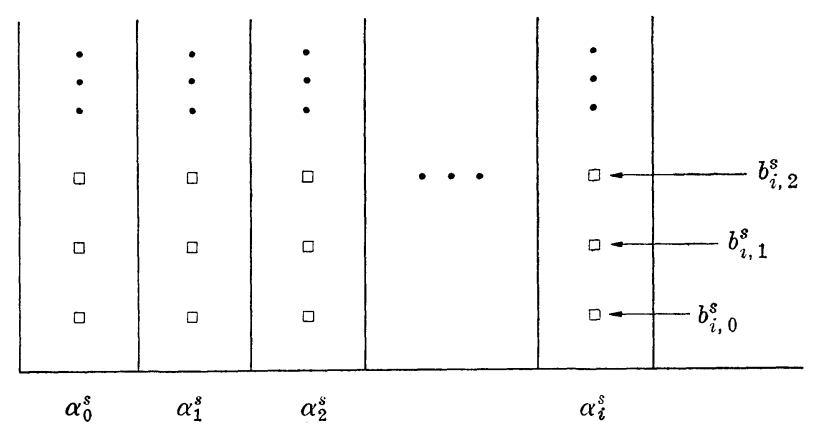

At the end of stage $s$, the windows in the $i$ th column will be occupied consecutively from the bottom up by $b_{i, 0}^{s}<b_{i, 1}^{s}<\cdots$ where

$$
\left\{b_{i, 0}^{s}, b_{i, 1}^{s}, \cdots\right\}=N_{i} \cap \bar{A}^{s}=\alpha_{i}^{s}
$$

and $A^{s}$ is the set of elements enumerated into $A$ by the end of stage s. Thus the windows give us a picture of the complement of $A^{s}$ at the end of stage $s$. Then during stage $s+1$, certain elements from 
the columns will be put into $A^{s+1}$ and the elements left in each column will drop down to fill in any vacant windows. We shall ensure that for each stage $s>0, A^{s} \cap N_{i}$ will be finite so that $\alpha_{i}^{s}$ will be infinite and every window will be occupied. For $s>0, A^{s}$ will always be an infinite recursive set.

We will meet three sets of requirements in the course of the construction. To ensure that each $\alpha_{i}$ is bi-dense, we must meet the following, requirements.

$$
D(i, n): \alpha_{i} \cap I_{n} \neq \varnothing \text {. }
$$

We will employ a set of markers $\Delta(i, n)$. At stage $s, \Delta(i, n)$ will rest on an $x \in \alpha_{i}^{s} \cap I_{n}$. Then for the sake of requirement $D(i, n)$ we will try to keep the element marked by $\Delta(i, n)$ out of $A$. If we are successful for all $i$ and $n$, then each $\alpha_{i}$ will be dense in $Q$ and hence each $\alpha_{i}$ will be bi-dense in $Q$ since $\bar{\alpha}_{i} \supseteqq \bigcup_{i \neq j} N_{j}$.

To ensure that condition (b) is satisfied by the $\alpha_{i}$ 's, we will meet the following set of requirements.

$Q(n): \varphi_{n} \uparrow \bar{A}$ is a $1-1$ map from $\bar{A}$ into $\bar{A}$ only if $\{a \in \bar{A} \mid a \neq \varphi(a)\}$ is finite. Suppose there is a recursive set $R \subseteq N$ and a partial recursive function $\varphi_{e}$ such that $\varphi_{e} \uparrow \mathrm{U}_{i \in R} \alpha_{i}$ is a $1-1$ map from $\bigcup_{i \in R} \alpha_{i}$ into $\bigcup_{i \in R} \alpha_{i}$ and $\left\{a \in \bigcup_{i \in R} \alpha_{i} \mid a \neq \varphi_{e}(a)\right\}$ is infinite. Let $\varphi_{n}$ be the recursive function defined by

$$
\varphi_{n}(x)= \begin{cases}\varphi_{e}(x) & \text { if } x \in \bigcup_{i \in R} N_{i} \text { and } x \in \delta \varphi_{e} \\ x & \text { if } x \in \bigcup_{i \in R} N_{i}=\bigcup_{i \in \bar{R}} N_{i} \\ \text { undefined otherwise } .\end{cases}
$$

Then $\varphi_{n}$ would violate requirement $Q(n)$. Thus if we meet all the requirements $Q(n)$, condition (b) will automatically follow.

The strategy to meet requirement $Q(n)$ at stage $s+1$ will be to try to find an $x \in \bar{A}^{s}$ such that $\varphi_{n}^{s}(x) \downarrow$ and $\varphi_{n}(x) \neq x$ and then put $\varphi_{n}(x)$ into $A^{s+1}$, put a marker $\lambda(n)$ on $x$, and then try to keep $x$ out of $A$. If $x \in \bar{A}$, then $x$ will witness that $\varphi_{n}(\bar{A}) \nsubseteq \bar{A}$. However, there may be two reasons why we cannot put $\varphi_{n}(x)$ into $A^{s+1}$. The first reason is that $\varphi_{n}(x)$ may already have another marker on it which means we want to keep $\varphi_{n}(x)$ out of $A$ for the sake of some other requirement. Thus, we must put a priority ranking on our list of requirements. We shall ensure that requirements with higher priority than $Q(n)$ restrict only finitely many elements from being put into $A$ so that if $\varphi_{n} \uparrow \bar{A}$ is $1-1$ and $\left\{a \in \bar{A} \mid a \neq \varphi_{n}(\alpha)\right\}$ is really infinite, we will be able to find a pair $\left(x, \varphi_{n}(x)\right)$ for which $\varphi_{n}(x)$ is never restricted by higher priority requirements. Then we will be able to put $\varphi_{n}(x)$ into $A$ and keep $x$ out of $A$. The second reason is that 
to ensure each $\alpha_{i} \leqq_{T} \beta$, we use a Yates permitting argument which puts some restrictions on which $b_{i, n}^{s}$ can be put into $A^{s+1}$. Thus it is also possible that $\varphi_{n}(x)$ is not 'permitted' to be put into $A^{s+1}$. In such a case, we shall place a $\lambda(n)$ marker on $x$ and try to keep $x$ out of $A$ in the hope that sometime later we will be permitted to put $\varphi_{n}(x)$ into $A$. We say requirement $Q(n)$ is satisfied at stage $s$ if there is an $x \in \bar{A}^{s}$ with a $\lambda(n)$ marker on it such that $\varphi_{n}^{s}(x) \downarrow$ and $\varphi_{n}^{s}(x) \in A^{s}$.

To ensure that each $\alpha_{i}$ has property (c), we must meet the following set of requirements.

$$
R(i, n): \quad \text { If } \alpha_{i} \leqq \delta \varphi_{n} \text { and } \varphi_{n} \uparrow \alpha_{i} \text { is } 1-1 \text {, then } \rho_{n}\left(\alpha_{i}\right) \nsubseteq \bigcup_{i \neq j} \alpha_{j}
$$

The requirements $R(i, n)$ have basically the same character as the requirements $Q(n)$. The strategy to meet requirement $R(i, n)$ at stage $s+1$ is to try to find an $x \in \alpha_{i}^{s}$ such that $\varphi_{n}^{s}(x) \downarrow$ and $x \neq \varphi_{n}^{s}(x)$ and either we can put $\varphi_{n}(x)$ into $A^{s+1}$ or $\varphi_{n}(x) \in N_{i}$. Then we put $\varphi_{n}(x)$ into $A^{s+1}$, if possible, and place a $\Gamma(i, n)$ marker on $x$ and try to keep $x$ out of $A$. If $x \in \bar{A}$, then $x \in \alpha_{i}$ and $x$ will witness that $\varphi_{n}\left(\alpha_{i}\right) \nsubseteq \bigcup_{i \neq j} \alpha_{j}$. Again the same type of restrictions as described above can restrict us from placing $\varphi_{n}(x)$ into $A^{s+1}$. We say that requirement $R(i, n)$ is satisfied at stage $s$ if there is an $x \in \overline{A^{s}}$ with a $\Gamma(i, n)$ marker on it such that $\varphi_{n}^{s}(x) \downarrow$ and $\varphi_{n}(x) \in A^{s} \cup N_{i}$.

It is clear that $\alpha_{i} \leqq_{T} A$ for each $i$. Thus to ensure that each $\alpha_{i} \leqq_{T} \beta$, we shall ensure that $A \leqq_{T} \beta$, using a Yates permitting argument where $b_{i, n}^{s}$ is allowed to be put into $A^{s}$ only if $\max (i, n) \geqq$ $f(s)$. Finally to force $\alpha_{i} \geqq_{T} \beta$, we shall use a coding argument where at each stage $s$ either $b_{i, f(s)}^{s}$ or $b_{i, f(s)+1}^{s}$ will be put into $A^{s+1}$ for each $i$. Thus at each stage $s>0, A^{s}$ will be an infinite but recursive set.

We make the following priority ranking of requirements:

$$
D(c(0), r(0)), Q(0), R(c(0), r(0)), D(c(1), r(1)), Q(1), R(c(1), r(1)), \cdots .
$$

(That is, $D(c(0), r(0))$ has highest priority, $Q(0)$ has the second highest priority, and so on.)

Only finitely many markers will be placed on elements at any given stage $s$. We assume we have infinitely many $\Delta(i, n), \lambda(n)$, and $\Gamma(i, n)$ markers at our disposal and if at stage $s+1$ we place a marker $\Phi$ on an $x \in \overline{A^{s}}$ such that at stage $s, x$ was unmarked or had a marker different form $\Phi$ on it, then $\Phi$ has never been used at any previous stage. If an $x \in \bar{A}^{s}$ drops to a lower window at stage $s+1$, the marker on $x$, if any, will stay with $x$ unless specifically stated otherwise. If an $x \in \overline{A^{s}}$ is put into $A^{s+1}$, then we automatically remove any marker on $x$. We say a marker $\Phi$ is active at stage $s$ if it rests 
on an $x \in \overline{A^{s}}$ and $\Phi$ is inactive otherwise. For simplicity, each $x$ will have at most one marker on it at any stage $s$. It will be possible for several markers of the same type to be active at a stage $s$. We say a marker $\Phi_{1}$ has higher priority than marker $\Phi_{2}$ if $\Phi_{1}$ corresponds to a higher priority requirement than $\Phi_{2}$ does. Finally, we define $\mathscr{H}(\Delta(i, n), s)=\{x \mid x$ has a marker $\Phi$ on it at stage $s$ and $\Phi$ has higher priority than $\mathcal{U}(i, n)\} . \quad \mathscr{H}(\lambda(n), s)$ and $\mathscr{H}(\Gamma(i, n), s)$ are defined similarly.

\section{Construction.}

Stage 0. Let $A^{0}=\varnothing$. Put a marker $\Delta(c(0), r(0))$ on the least $x$ in $N_{c(0)} \cap I_{r(0)}$.

Stage $s+1$. Assume that $A^{s}$ is recursive and that at stage $s$

(a) $A^{s} \cap N_{i}$ is finite for each $i$,

(b) only finitely many markers are active and no $x \in \overline{A^{s}}$ has more than one marker on it,

(c) for all $j \leqq s$, exactly one $\Delta(c(j), r(j))$ marker is active and it rests on an $x \in N_{c(j)} \cap I_{r(j)}$,

(d) a $\lambda(n)$ marker rests on $x$ only if $\varphi_{n}^{s}(x) \downarrow$ and $x \neq \varphi_{n}(x)$ and a $\Gamma(i, n)$ marker rests on $x$ only if $\varphi_{n}^{s}(x) \downarrow, x \neq \varphi_{n}(x)$, and $x \in \alpha_{i}^{s}$,

(e) if requirement $Q(j)(R(j, n))$ is satisfied, then exactly one $\lambda(j)(\Gamma(j, n))$ marker is active.

Look for a $j \leqq s+1$ such that at stage $s$ either

(1) $Q(j)$ is not satisfied and there is an $x \leqq s+1$ such that $x \in \bar{A}^{s}-\mathscr{H}(\lambda(j), s), \varphi_{j}^{s+1}(x) \downarrow, x \neq \varphi_{j}(x)$, and either $x \notin\left\{b_{i, f(s)}^{s}, b_{i, f(s)+1}^{s}\right\}$ for any $i$ or if $x \in\left\{b_{i, f(s)}^{s}, b_{i, f(s)+1}^{s}\right\}$, then $y \in\left\{b_{i, f(s)}^{s}, b_{i, f(s)+1}^{s}\right\}-\{x\}$ implies $y \notin \mathscr{H}(\lambda(j), s)$, and moreover either

(1A) $\varphi_{j}(x) \notin\left\{b_{i, n}^{s} \mid \max (i, n)<f(s)\right\} \cup \mathscr{H}(\lambda(j), s)$ or

(1B) $\varphi_{j}(x) \in\left\{b_{i, n}^{s} \mid \max (i, n)<f(s)\right\}-\mathscr{H}(\lambda(j), s)$ and if $b_{i, n}^{s}=\varphi_{j}(x)$, then for all $b_{e, k}^{s}=\varphi_{j}(y)$, where $y$ has a $\lambda(n)$ marker on it, $\max (i, n)>$ $\max (e, k)+1$,

(2) Condition (1) fails and $R(c(j), r(j))$ is not satisfied and there is an $x \leqq s+1$ such that $x \notin \overline{A^{s}}-\mathscr{H}(\Gamma(c(j), r(j)), s), \varphi_{r(j)}^{s+1}(x) \downarrow, x \neq$ $\varphi_{r(j)}(x)$, and either $x \notin\left\{b_{i, f(s)}^{s}, b_{i, f(s)+1}^{s}\right\}$ for any $i$ or if $x \in\left\{b_{i, f(s)}^{s}, b_{i, f(s)+1}^{s}\right\}$, then $y \in\left\{b_{i, f(s)}^{s}, b_{i, f(s)+1}^{s}\right\}-\{x\}$ implies $y \notin \mathscr{H}(\Gamma(c(j), r(j)), s)$, and moreover either

or

(2A) $\varphi_{r^{(j)}}(x) \notin\left[\left\{b_{i, n}^{s} \mid \max (i, n)<f(s)\right\} \cup \mathscr{C}(\Gamma(c(j), r(j)), s)\right]-N_{c(j)}$

(2B) $\varphi_{r(j)}(x) \in\left\{b_{i, n}^{s} \mid \max (i, n)<f(s)\right\}-\left(\mathscr{H}(\Gamma(c(j), r(j), s)) \cup N_{c(j)}\right)$ and if $b_{i, n}^{s}=\varphi_{r(j)}(x)$, then for all $b_{e, k}^{s}=\varphi_{r(j)}(y)$ where $y$ has a $\Gamma(c(j)$, $r(j))$ marker on it, $\max (i, n)>\max (e, k)+1$.

If there is no such $j$, go to Case 0 . If there is such a $j$, let $e(s+1)$ 
be the least such $j$ and go to Case 1 if $e(s+1)$ satisfies condition (1) and go to Case 2 otherwise.

Case 0. For each $i$, consider the pair $x_{i}=b_{i, f(s)}^{s}$ and $y_{i}=b_{i, f(s)+1}^{s}$ and the markers that currently rest on $x_{i}$ and $y_{i}$, if any. If $x_{i}$ is not marked, put $x_{i}$ into $A^{s+1}$. If $x_{i}$ is marked and $y_{i}$ is not marked, put $y_{i}$ into $A^{s+1}$. Otherwise, suppose marker $\Phi_{1}$ rests on $x_{i}$ and marker $\Phi_{2}$ rests on $y_{i}$. If $\Phi_{2}$ has higher priority than $\Phi_{1}$, put $x_{i}$ into $A^{s+1}$ and if $\Phi_{1}$ has higher priority than $\Phi_{2}$, put $y_{i}$ into $A^{s+1}$. If $\Phi_{1}$ and $\Phi_{2}$ have the same priority, then $\Phi_{1}$ and $\Phi_{2}$ must either be $\lambda(n)$ markers or $\Gamma(i, n)$ markers for some $n$. In such a case, let $b_{a, m}^{s}=$ $\varphi_{n}\left(x_{i}\right)$ and $b_{c, k}^{s}=\varphi_{n}\left(y_{i}\right)$. Put $x_{i}$ into $A^{s+1}$ if $\varphi_{n}\left(x_{i}\right)$ is in

$$
\mathscr{H}(\lambda(n), s)(\mathscr{H}(\Gamma(i, n), s))
$$

and $\varphi_{n}\left(y_{i}\right)$ is not and put $y_{i}$ in $A^{s+1}$ if $\varphi_{n}\left(y_{i}\right)$ is in

$$
\mathscr{H}(\lambda(n), s)(\mathscr{H}(\Gamma(i, n), s))
$$

and $\varphi_{n}\left(x_{i}\right)$ is not. Finally, if $\varphi_{n}\left(x_{i}\right), \varphi_{n}\left(y_{i}\right) \in \mathscr{H}(\lambda(n), s)(\mathscr{H}(\Gamma(i, n), s))$ or $\varphi_{n}\left(x_{i}\right), \varphi_{n}\left(y_{i}\right) \notin \mathscr{H}(\lambda(n), s)(\mathscr{H}(\Gamma(i, n), s))$, put $x_{i}$ into $A^{s+1}$ if $\max (a, m) \leqq \max (c, k)$ and put $y_{i}$ into $A^{s+1}$ if $\max (a, m)>\max (c, k)$.

Case 1. Let $e=e(s+1)$ and $z$ be the least $x$ corresponding to $e$ such that $\varphi_{e}(x)$ satisfies condition (1A) if there is a pair $\left(y, \varphi_{e}(y)\right)$ satisfying condition (1A) or $\varphi_{e}(x)$ satisfies condition (1B) if there is no pair $\left(y, \varphi_{e}(y)\right)$ satisfying condition $(1 \mathrm{~A})$.

(A) If $\varphi_{e}(z)$ satisfies condition (1A), place a new $\lambda(e)$ marker on $z$ and remove any marker that was on $z$ at stage $s$ and all $\lambda(e)$ markers that were active at stage $s$. Then put $\varphi_{e}(z)$ into $A^{s+1}$ if it is not already in $A^{s}$. For each $i$, also put either $b_{i, f(s)}^{s}$ or $b_{i, f(s)+1}^{s}$ into $A^{s+1}$ according to the instructions in Case 0. (Note: our choice of $z$ ensures that $z \notin A^{s+1}$ so that requirement $Q_{n}$ will be satisfied at stage $s+1$.)

(B) If $\varphi_{e}(z)$ satisfies condition (1B), place a new $\lambda(e)$ marker on $z$ and remove any marker that was on $z$ at stage $s$. Then, for each $i$, put either $b_{i, f(s)}^{s}$ or $b_{i, f(s)+1}^{s}$ into $A^{s+1}$ according to the instructions in case 0 .

Case 2. Let $e=e(s+1)$ and let $z$ be the least $x$ corresponding to $e$ such that $\varphi_{r(e)}(x)$ satisfies condition (2A) if there is pair $\left(y, \varphi_{r(e)}(y)\right)$ satisfying condition $(2 \mathrm{~A})$ or $\varphi_{r(e)}(x)$ satisfies condition (2B) if there is no pair $\left(y, \varphi_{r(e)}(y)\right)$ satisfying condition (2A).

(A) If $\varphi_{r(e)}(z)$ satisfies condition (2A), place a new $\Gamma(c(e), r(e))$ marker on $z$ and remove any marker that was on $z$ at stage $s$ and 
all $\Gamma(c(e), r(e))$ markers that were active at stage $s$. Then put $\varphi_{e}(z)$ into $A^{s+1}$ if $\varphi_{e}(z) \notin \mathscr{C}(\Gamma(c(e), r(e), s)) \cup N_{c(e)} \cup A^{s}$. For each $i$, put either $b_{i, f(s)}^{s}$ or $b_{i, f(s)+1}^{s}$ into $A^{s+1}$ according to the instructions in Case 0 . (Note: our choice of $z$ ensures that $z \notin A^{s+1}$ so that requirement $R(c(e), r(e))$ will be satisfied at stage $s+1$.)

(B) If $\varphi_{r(e)}(z)$ satisfies condition (2B), place a new $\Gamma(c(e), r(e))$ marker on $z$ and remove any marker that was on $z$ at stage $s$. Then for each $i$, put either $b_{i, f(s)}^{s}$ or $b_{i, f(s)+1}^{s}$ into $A^{s+1}$ according to the instructions in case 0 .

This completes the definition of $A^{s+1}$. It is possible that for some $j$ and $n$, requirement $Q(n)(R(j, n))$ was not satisfied at stage $s$ but there is now some $x \in \overline{A^{s+1}}$ with a $\lambda(n)(\Gamma(j, n))$ marker on it and $\varphi_{n}(x) \in A^{s+1}$ because $\varphi_{n}(x) \in \bigcup_{i}\left\{b_{i, f(s)}^{s}, b_{i, f(s)+1}^{s}\right\}$ and $\varphi_{n}(x)$ was forced into $A^{s+1}$. In such a case, we keep the $\lambda(n)(\Gamma(j, n))$ marker on the least such $x$ and remove all other $\lambda(n)(\Gamma(j, n))$ markers that were active at stage $s$. Finally, some of the $\Delta(c(i), r(i))$ markers for $i \leqq s$ may have been removed. Inductively we place new $\Delta(c(i), r(i))$ markers for $i \leqq s+1$ as follows: having placed $\Delta(c(j), r(j))$ markers for $j<i$, place $\Delta(c(i), r(i))$ on the least $x \in \alpha_{c(i)}^{s+1} \cap I_{r(i)}$ which is unmarked if $\Delta(c(i), r(i))$ was removed during stage $s+1$ and otherwise leave $\Delta(c(i), r(i))$ where it is. (This is possible since $A^{s+1} \cap N_{c(i)}$ is finite and $N_{c(i)}$ is dense in $\left.Q.\right)$

This completes the description of stage $s+1$. It is easy to check that each stage is completely effective and that conditions (a)-(e) hold at each stage. We let $A=\mathrm{U}_{s} A^{s}$ so that $A$ is r.e. We now prove a sequence of lemmas that will complete the proof of the theorem.

LemMa 1. For all $i$ and $n, \lim _{s} b_{i, n}^{s}$ exists.

Proof. $b_{i, n}^{s} \neq b_{i, n}^{s+1}$ only if $f(s) \leqq \max (i, n)$. Since $f(s) \leqq \max (i, n)$ only finitely often, $\lim _{s} b_{i, n}^{s}$ exists.

\section{LEMMA 2. $A \leqq_{T} \beta$.}

Proof. It follows from our construction that for all $x, x=b_{i, n}^{s}$ and $x=b_{j, k}^{s+1}$ only if $i=j$ and $k \leqq n$. Thus to decide if $x \in A$, first find $i$ and $n$ such that $x=b_{i, n}^{0}$. Then recursively in $\beta$, find a stage $t$ such that $\forall s(s \geqq t \rightarrow f(s)>\max (i, n))$. Since for any $j$ and $k, b_{j, k}^{s} \neq$ $b_{j, k}^{s+1}$ only if $f(s) \leqq \max (j, k)$, it follows that $\forall k \forall s(k \leqq n \& s \geqq t \rightarrow$ $\left.b_{i, k}^{s}=b_{i, k}\right)$. Thus $x \in A$ iff $x \notin\left\{b_{i, 0}^{t}, \cdots, b_{i, n}^{t}\right\}=\left\{b_{i, 0}, \cdots, b_{i, n}\right\}$. Therefore, $A \leqq{ }_{T} \beta$. 
Since for each $i, \alpha_{i}=\bar{A} \cap N_{i} \leqq_{T} A$, we have that $\alpha_{i} \leqq_{T} \beta$. Thus to prove that for each $i, \alpha_{i} \equiv_{T} \beta$, we need only show that for each $i, \beta \leqq{ }_{T} \alpha_{i}$.

Lemma 3. For each $i, \beta \leqq{ }_{T} \alpha_{i}$.

Proof. We note that for each $i, \alpha_{i}=\left\{b_{i, 0}, b_{i, 1}, \cdots\right\}$ and $b_{i, 0}<$ $b_{i, 1}<\cdots$ since for all $s, b_{i, 0}^{s}<b_{i, 1}^{s}<\cdots$. To decide if $x \in \beta$, first find, recursively in $\alpha_{i}$, a stage $t$ such that $\forall k\left(k \leqq x+1 \rightarrow b_{j, k}^{t}=b_{i, k}\right)$. Since for any pair $(j, n)$ and stage $s, b_{j, n}^{s} \neq b_{j, n}^{s+1}$ only if there is a $k \leqq n$ such that $b_{j, k}^{s} \in A^{s+1}$, it follows that $\forall s \forall k(k \leqq x+1 \& s \geqq t \rightarrow$ $\left.b_{i, k}^{s}=b_{i, k}\right)$. Since at each stage $s+1$, we put either $b_{i, f(s)}^{s}$ or $b_{i, f(s)+1}^{s}$ into $A^{s+1}$, it follows that $\forall s(s \geqq t \rightarrow f(s)>x)$. Thus, $x \in \beta$ if $x \in \beta^{t}$ and hence $\alpha_{i} \geqq_{T} \beta$.

LEMMA 4. For each $n$, the requirements $D(c(n), r(n)), Q(n)$, and $R(c(n), r(n))$ are met.

Proof. We proceed by induction. Fix $n \geqq 0$ and assume that for all $i<n$, the requirements $D(c(i), r(i)), Q(i)$, and $R(c(i), r(i))$ are met and there is a stage $t>n$ and an integer $p$ such that: (a) For all $s \geqq t$ and $j<n$, no new $\Delta(c(j), r(j)), \lambda(j)$, or $\Gamma(c(j), r(j))$ marker becomes active or old $\Delta(c(j), r(j)), \lambda(j)$, or $\Gamma(c(j), r(j))$ marker is removed at stage $s$, (b) If $b_{i, k}^{t} \in \mathscr{C}(\Delta(c(n), r(n)), t)$, then $\max (i, k)<$ $p$, (c) $\forall s(s \geqq t \rightarrow f(s)>p)$, and (d) $\forall s(s \geqq t \rightarrow e(s) \geqq n)$. Thus by stage $t$ all $\Delta(c(i), r(i)), \lambda(i)$, and $\Gamma(c(i), r(i))$ markers with $i<n$ rest on elements that never move after stage $t$.

First, we consider the requirement $D(c(n), r(n))$. Suppose that at stage $t+1, \Delta(c(n), r(n))$ rests on $x \in \alpha_{c(n)}^{t+1} \cap I_{r(n)}$. We claim that for all $s \geqq t+1, \Delta(c(n), r(n))$ rests on $x$ and thus $x \in \alpha_{c(n)} \cap I_{r(n)}$. For assume $s \geqq t+1, x=b_{c(n), j}^{s}$ for some $j$, and $\Delta(c(n), r(n))$ rests on $x$ at stage $s$. Then at stage $s+1$, if $e(s+1)$ is defined, $e(s+1) \geqq n$ so that $x \neq z, x \neq \varphi_{e(s+1)}(z)$ for $z$ as defined in Case 1 and $x \neq z$, $x \neq \varphi_{r(e(s+1))}(z)$ for $z$ as defined in Case 2. Thus the only way $x$ could be put into $A^{s+1}$ is if $j \in\{f(s), f(s)+1\}$. By our choice of $t, f(s)>p$ and thus the $y \in\left\{b_{c(n), f(s)}^{s}, b_{c(n), f(s)+1}^{s}\right\}-\{x\}$ is not in $\mathscr{C}(\Delta(c(n), r(n)), s)$. Hence $\Delta(c(n), r(n))$ must have a higher priority than the marker on $y$, if any, and hence $y$ and not $x$ would be placed into $A^{s+1}$. It follows that after stage $t+1$ no new $\Delta(c(n), r(n))$ marker is ever introduced so that $\forall s(s \geqq t+1 \rightarrow \mathscr{H}(\lambda(n), s)=\mathscr{H}(\lambda(n), s+1))$. Let $x=b_{c(n), k}$ and choose $t_{1}>t$ and $p_{1}>p$ such that $\max (c(n), k)<p_{1}$ and $\forall s\left(s \geqq t_{1} \rightarrow f(s) \geqq p_{1}\right)$.

Now consider the requirement $Q(n)$. First we show that if $Q(n)$ is ever satisfied for some $s>t_{1}$, then requirement $Q(n)$ is met and 
there is a stage $t_{2}$ and an integer $p_{2}$ such that $\left(a^{\prime}\right)$ for all $s \geqq t_{2}$, $i \leqq n$, and $j<n$, no new $\Delta(c(i), r(i)), \lambda(i)$, or $\Gamma(c(j), r(j))$ marker becomes active or old $\Delta(c(i), r(i)), \lambda(i)$, or $\Gamma(c(j), r(j))$ marker is removed at stage $s,\left(\mathrm{~b}^{\prime}\right)$ if $b_{i, k}^{t_{2}} \in \mathscr{H}\left(\Gamma(c(n), r(n)), t_{2}\right)$, then $\max (i, k)<p_{2}, \quad\left(\mathrm{c}^{\prime}\right)$ $\forall s\left(s \geqq t_{2} \rightarrow f(s)>p_{2}\right)$, and $\left(\mathrm{d}^{\prime}\right) \forall s\left(s \geqq t_{2} \rightarrow e(s)>n \vee(e(s)=n\right.$ and we are in Case 2 at stage $s)$ ).

Suppose $u>t_{1}$ and $Q(n)$ is satisfied at stage $u$. Thus there is an $x \in \bar{A}^{u}$ with a $\lambda(n)$ marker on it such that $\varphi_{n}^{u}(x) \downarrow$ and $\varphi_{n}(x) \in A^{u}$. We claim that $x$ can never be put into $A$ and the marker $\lambda(n)$ is never removed from $x$ so that $Q(n)$ remains satisfied for all $s \geqq u$. For suppose $s \geqq u, x \in \overline{A^{s}}$, and $x$ has $a \lambda(n)$ marker on it so that $Q(n)$ is satisfied at stage $s$. If $e(s+1)$ is defined, then either $e(s+1)>n$ or $e(s+1)=n$ and we are in Case 2 at stage $s+1$. Hence marker $\lambda(n)$ is not removed from $x$ for the sake of a higher priority requirement and thus the only way $x$ can be put into $A^{s+1}$ is if $x=b_{i, k}^{s}$ for some $k \in\{f(s), f(s)+1\}$. By our choice of $s \geqq u>t_{1}, f(s)>p_{1}$ and thus the $y \in\left\{b_{i, f(s)}^{s}, b_{i, f(s)+1}^{s}\right\}-\{x\}$ is not in $\mathscr{C}(\lambda(n), s)$. Thus $\lambda(n)$ must have a higher priority than the marker on $y$, if any, and hence $y$ and not $x$ would be placed into $A^{s+1}$. Thus it follows that after stage $u$, no new $\lambda(n)$ marker is ever introduced so that $\forall s(s \geqq u \rightarrow$ $\mathscr{H}(\Gamma(c(n), r(n)), s)=\mathscr{H}(\Gamma(c(n), r(n)), u)$. We have also shown that $x \in \bar{A}$ so that if $x=b_{i, k}$ we need only choose $p_{2}>\max \left(p_{1}, i, k\right)$ and $t_{2} \geqq u$ such that $\forall s\left(s \geqq t_{2} \rightarrow f(s) \geqq p_{2}\right.$ and $\left.b_{i, k}^{s}=b_{i, k}\right)$ and then $p_{2}$ and $t_{2}$ will satisfy conditions $\left(a^{\prime}\right)-\left(d^{\prime}\right)$.

Now consider the case where there is no stage $s \geqq t_{1}$ such that $Q(n)$ is satisfied at stage $s$. We claim that under this assumption, there are only finitely many $s \geqq t_{1}$ such that $e(s)=n$ and we are in Case 1 at stage $s$. For suppose there are infinitely many such $s$; we will show that $\beta$ is recursive, contradicting our choice of $\beta$. First we shall prove by induction that if $u \geqq t_{1}$ and there is an $x \in \bar{A}^{u}$ with a $\lambda(n)$ marker on it at stage $u$ such that $\varphi_{n}(x)=b_{i, k}^{u} \notin \mathscr{H}(\lambda(n), u)$, then for all $s \geqq u$, there is a $y \in \overline{A^{s}}$ with a $\lambda(n)$ marker on it at stage $s$ such that $\varphi_{n}(y)=b_{j, l}^{s} \notin \mathscr{C}(\lambda(n), s)$ and $\max (j, l) \geqq \max (i, k)$. Let $s \geqq u$ and assume there is a $y$ with the properties above. Now either $y \notin\left\{b_{i, f(s)}^{s}, b_{i, f(s)+1}^{s}\right\}$ for any $i$ or if $y \in\left\{b_{i, f(s)}^{s}, b_{i, f(s)+1}^{s}\right\}$, then since $f(s)>p_{1}$ the $y^{\prime} \in\left\{b_{i, f(s)}^{s}, b_{i, f(s)+1}^{s}\right\}-\{y\}$ does not have a higher priority marker than $\lambda(n)$ on it. Thus at stage $s+1$, it cannot be that $f(s) \leqq \max (j, l)$ because then $\left(y, \varphi_{n}(y)\right)$ would be a pair which could satisfy $Q(n)$ and hence our choice of $s \geqq u>t_{1}$ would imply that $e(s+1)=n$ and that we are in Case 1 at stage $s+1$. In such a case, $Q(n)$ would be satisfied at stage $s+1$ which we assumed is not the case. Thus $f(s)>\max (j, l)$ and $\varphi_{n}(y)=b_{j, l}^{s}=b_{j, l}^{s+1}$. Since $e(s+1) \geqq n$, it follows that if $e(s+1)$ is defined, then $y \neq z, y \neq$ 
$\varphi_{e(s+1)}(z)$ if we are in Case 1 and $y \neq z, y \neq \varphi_{r(e(s+1))}(z)$ if we are in Case 2 at stage $s+1$. Thus the only way $y$ could be put into $A^{s+1}$ is if $y \in\left\{b_{i, f(s)}^{s}, b_{i, f(s)+1}^{s}\right\}$ for some $i$.

Since $f(s)>p_{1}, \lambda(n)$ is the highest priority marker that could rest on either $b_{i, f(s)}^{s}$ or $b_{i, f(s)+1}^{s}$. Thus the only way $y$ could be put into $A^{s+1}$ is if the $y^{\prime} \in\left\{b_{i, f(s)}^{s}, b_{i, f(s)+1}^{s}\right\}-\{y\}$ also has a $\lambda(n)$ marker on it and $\varphi_{n}\left(y^{\prime}\right)=b_{a, m}^{s} \notin \mathscr{H}(\lambda(n), s)$ and $\max (a, m) \geqq \max (j, l)$. Moreover, it must be the case that $f(s)>\max (a, m)$ and hence $b_{a, m}^{s}=b_{a, m}^{s+1}$. Thus either $y$ or $y^{\prime}$ is in $\overline{A^{s+1}}$ and has a $\lambda(n)$ marker on it at stage $s+1$. Since $\mathscr{H}(\lambda(n), s)=\mathscr{H}(\lambda(n), s+1)$, we can conclude that $\varphi_{n}(y), \varphi_{n}\left(y^{\prime}\right) \in \overline{A^{s+1}}-\mathscr{H}(\lambda(n), s+1)$ and hence either $\left(y, \varphi_{n}(y)\right)$ or $\left(y^{\prime}, \varphi_{n}\left(y^{\prime}\right)\right)$ satisfies the required properties at stage $s+1$.

We define $l^{s}=\max \left(\left\{\max (j, k) \mid \exists y\left(y \in \bar{A}^{s}\right.\right.\right.$ and $y$ has a $\lambda(n)$ marker on it at stage $s$ and $\left.\left.\varphi_{n}(y)=b_{j, k}^{s} \notin \mathscr{H}(\lambda(n), s)\right\}\right)$. The immediately preceding induction proved that if $s \geqq t_{1}$ and $l^{s}$ is defined, then $f(s)>l^{s}$ and $l^{s+1}$ is defined and $l^{s+1} \geqq l^{s}$. Thus if $s \geqq t_{1}$ and $l^{s}$ is defined, then $\forall u\left(u \geqq s \rightarrow f(u)>l^{u} \geqq l^{s}\right)$. Now suppose $s_{1} \geqq t, e\left(s_{1}\right)=n$, and we are in Case 1 at stage $s_{1}$. If $z$ is defined as in Case 1 , then $\varphi_{n}(z)$ must satisfy clause (1B) of the definition of $e\left(s_{1}\right)$ so that $\varphi_{n}(z) \notin$ $\mathscr{H}\left(\lambda(n), s_{1}-1\right)=\mathscr{C}\left(\lambda(n), s_{1}\right)$. Thus $l^{s_{1}}$ must be defined. If $s_{2}>s_{1}$ and $e\left(s_{2}\right)=n$ and we are in Case 1 at $s_{2}$, then let $z^{*}$ denote the $z$ defined in Case 1 at stage $s_{2}$. We know $l^{s_{2}-1}$ is defined, $l^{s_{2}-1} \geqq l^{s_{1}}$, and $\varphi_{n}\left(z^{*}\right)$ must satisfy clause (1B) of the definition of $e\left(s_{2}\right)$; thus $\varphi_{n}\left(z^{*}\right)=$ $b_{a, m}^{s} \notin \mathscr{C}\left(\lambda(n), s_{2}-1\right)$ and $\max (\alpha, m)>l^{s_{2}-1}+1$. Then $z^{*}$ has a $\lambda(n)$ marker on it at stage $s_{2}$ and $\varphi_{n}\left(z^{*}\right)=b_{e, g}^{s_{2}}$ where $\max (e, g)>l^{s_{2}-1}$ since no more than one element is removed from any one column. Thus $l^{s_{2}}>l^{s_{2}-1}$. It follows that if there are infinitely many $s \geqq t_{1}$ such that $e(s)=n$ and we are in Case 1 at stage $s$, then we can find a recursive sequence of stages $t_{1} \leqq s_{1}<s_{2}<\cdots$ such that $l^{s_{1}}<l^{s_{2}}<\cdots$. But the existence of such a sequence would imply that $\beta$ is recursive. For to decide if $x \in \beta$, we need only find a stage $s_{i}$ such that $l^{s_{i}} \geqq x$ and then we know $x \in \beta$ iff $x \in \beta^{s_{i}}$ since $\forall s\left(s>s_{i} \rightarrow f(s)>l^{s_{i}}\right)$.

Thus we have shown that if $Q(n)$ is never satisfied at any stage $s \geqq t_{1}$, then $e(s)=n$ and we are in Case 1 at stage $s$ for only finitely many $s \geqq t_{2}$. Since new $\lambda(n)$ markers can be introduced only at stage $s^{\prime}$ where $e(s)=n$ and we are in Case 1 at stage $s$, it follows that there are $t_{2}$ and $p_{2}$ which satisfy conditions $\left(\mathrm{a}^{\prime}\right)-\left(\mathrm{d}^{\prime}\right)$. However we must still check that if $Q(n)$ is never satisfied for any $s \geqq t_{1}$, then requirement $Q(n)$ is met. Suppose requirement $Q(n)$ fails. Thus $\bar{A} \cong \delta \varphi_{n}$ and $\varphi_{n} \uparrow \bar{A}$ is a $1-1$ map from $\bar{A}$ into itself and $\left\{a \in \bar{A} \mid a \neq \varphi_{n}(\alpha)\right\}$ is infinite. We have shown the existence of a stage $t_{2}$ such that for all $s \geqq t_{2}$ either $e(s)>n$ or $e(s)=n$ and we are in Case 2 at stage $s$. But consider stage $t_{2}$. Since $\mathscr{H}(\lambda(n), s)=\mathscr{H}\left(\lambda(n), t_{1}\right)$ 
for all $s \geqq t_{1}$, there must be an $x \in \bar{A}$ such that $x \neq \varphi_{n}(x)$ and $\varphi_{n}(x)=$ $b_{j, k} \notin \mathscr{K}\left(\lambda(n), t_{2}\right)$ and if $l^{t_{2}}$ is defined, then $\max (j, k)>l^{t_{2}}+1$. Now suppose $s>t_{2}$ is a stage such that $\varphi_{n}^{s}(x) \downarrow$. Then $\varphi_{n}^{s}(x)=b_{j, m}^{s}$ for some $m>k$ and either $x \notin\left\{b_{i, f(s)}^{s}, b_{i, f(s)+1}^{s}\right\}$ for any $i$ or if $x \in\left\{b_{i,(s)}^{s}, b_{i, f(s)+1}^{s}\right\}$, then since $f(s)>p_{1}$, the $y \in\left\{b_{i, f(s)}^{s}, b_{i, f(s)+1}^{s}\right\}-\{x\}$ does not have a higher priority marker than $\lambda(n)$ on it. Thus the pair $\left(x, \varphi_{n}(x)\right)$ would be candidates to satisfy Case 1 of the definition of $e(s)$ for $n$ unless $l^{s-1}$ is defined and $\max (j, m) \nless l^{s-1}+1$. Therefore, since our choice of $t_{2}$ precludes us from being in Case 1 with $e(s)=n$ at stage $s$, it must be the case that $\max (j, m) \nless l^{s-1}+1$. Now if $l^{t_{2}}$ was defined, then $l^{s-1}>l^{t_{2}}$. Thus we must conclude there is a stage $s^{\prime} \geqq t_{2}$ such that either $l^{s^{\prime}-1}$ was undefined and $l^{s^{\prime}}$ is defined or $l^{s^{\prime-1}}$ is defined and $l^{s^{\prime}}>l^{s^{\prime}-1}$. But both of these cases imply that we are in Case 1 with $e\left(s^{\prime}\right)=n$ a stage $s^{\prime}$ which contradicts our choice of $t_{2}$. Thus requirement $Q(n)$ must be met.

We have shown requirement $Q(n)$ must have been met and there are $t_{2}$ and $p_{2}$ satisfying conditions $\left(\mathbf{a}^{\prime}\right)-\left(\mathrm{d}^{\prime}\right)$. The argument for requirement $R(c(n), r(n))$ is almost exactly the same as the one for requirement $Q(n)$. Namely, we can show that if there is an $s \geqq t_{2}$ such that $R(c(n), r(n))$ is satisfied at stage $s$, then requirement $R(c(n), r(n))$ is met and there is a stage $t_{3}$ and an integer $p_{3}$ such that $\left(\mathrm{a}^{\prime \prime}\right)$ for all $s \geqq t_{3}$ and $j \leqq n$, no new $\Delta(c(j), r(j)), \lambda(j)$, or $\Gamma(c(j), r(j))$ marker becomes active or old $\Delta(c(j), r(j)), \lambda(j)$, or $\Gamma(c(j), r(j))$ marker is removed at stage $s$, $\left(b^{\prime \prime}\right)$ if $b_{i, k}^{t_{3}} \in \mathscr{H}\left(\Delta(c(n+1), r(n+1)), t_{3}\right)$, then $\max (i, k)<p_{3},\left(\mathrm{c}^{\prime \prime}\right) \forall s\left(s \geqq t_{3} \rightarrow f(s)>p_{3}\right)$, and $\left(\mathrm{d}^{\prime \prime}\right) \forall s\left(s \geqq t_{3} \rightarrow e(s) \geqq\right.$ $n+1)$. If there is no stage $s \geqq t_{3}$ such that $R(c(n), r(n))$ is satisfied at stage $s$, then we can argue that the assumption that there are infinitely many $s \geqq t_{3}$ such that we are in Case 2 with $e(s)=n$ at stage $s$ leads to the contradiction that $\beta$ is recursive. Hence there can be only finitely many $s$ such that we are in Case 2 with $e(s)=n$ at stage $s$ and thus there are $t_{3}$ and $p_{3}$ satisfying conditions $\left(a^{\prime \prime}\right)-\left(d^{\prime \prime}\right)$. Finally, we can argue that existence of $t_{3}$ and $p_{3}$ implies that requirement $R(c(n), r(n))$ is met. These arguments complete the induction step for $n$.

THEOREM 2. Let $\beta$ be any recursively enumerable set which is not recursive and let $P=\left(N, \leqq^{*}\right)$ be a recursive partial ordering. Then there is a collection co-r.e. bi-dense subsets of $Q$ with property $\mathscr{P}$, each Turing equivalent to $\beta$, such that under $\prec_{c}, \prec_{i}, \prec_{e}$, this collection is order isomorphic to $P$.

Proof. Since $P$ is a recursive partial ordering, $R_{i}=\left\{j \in N \mid i \leqq{ }^{*} j\right\}$ is a recursive set for each $i$. Let $M$ be a map from $N$ into the set of all subsets of $N$ defined by $M(i)=\mathrm{U}_{j \in R_{i}} \alpha_{j}$. It easily follows that 
for each $i, M(i)$ is a co-r.e. bi-dense subset of $Q$ which has property $\mathscr{P}$ and is Turing equivalent to $\beta$. We shall prove that $M$ is an order preserving map from $\left(N, \leqq^{*}\right)$ onto $\{M(i) \mid i \in N\}$ under either $\prec_{i}, \prec_{c},<_{e}$. First we show $M$ is $1-1$. If $M(i)=M(k)$, then it must be the case that $R_{i}=R_{k}$. Thus $i \in R_{k}=\{j \in N \mid j \leqq * k\}$ and hence $i \leqq * k$. Similarly $k \leqq * i$ so that $k=i$. Now suppose $i \leqq * k$ and $i \neq k$; we show that $M(i) \prec_{i} M(k), M(i) \prec_{c} M(k)$, and $M(i) \prec_{e} M(k)$. $R_{i}$ is strictly contained in $R_{k}$ since $k \in R_{k}-R_{i}$. Thus $M(i) \subset M(k)$. Moreover if $W=\bigcup_{j \in R_{i}} N_{j}$ and $\bar{W}=\bigcup_{j \in \bar{R}_{i}} N_{j}$ where $N_{j}$ are the sets defined in Theorem 1 , then $W$ and $\bar{W}$ are recursive sets. Also $W \cap M(k)=W \cap \bigcap_{j \in R_{k}} \alpha_{j}=\bigcap_{j \in R_{i}} \alpha_{j}=M(i)$ and $\bar{W} \cap M(k)=\bar{W} \cap \bigcup_{j \in R_{k}} \alpha_{j}=$ $\mathrm{U}_{j \in R_{k}-R_{i}} \alpha_{j}=M(k)-M(i)$. Thus $W$ and $\bar{W}$ witness that $M(i) \prec_{i} M(k)$. It follows immediately from the definitions of $<_{i},<_{c}$, and $<_{e}$ that $\forall \alpha, \beta \subseteq Q\left(\alpha \prec_{i} \beta \rightarrow \alpha \prec_{c} \beta \rightarrow \alpha \prec_{e} \beta\right)$. Thus we also have $M(i) \prec_{c} M(k)$ and $M(i) \prec_{e} M(k)$. Now suppose $i \not{ }^{*} k$. Thus $i \notin R_{k}$ so that $\alpha_{i} \cap M(k)=$ $\alpha_{i} \cap \bigcup_{j \in R_{k}} \alpha_{i}=\varnothing$. We claim that $M(i) \prec_{e} M(k)$. For if $M(i) \prec_{e} M(k)$. then there is a partial recursive function $\varphi$ such that $M(i) \leqq \delta \varphi$ and $\varphi \uparrow M(i)$ is a $1-1$ map from $M(i)$ into $M(k)$. But then $\alpha_{i} \leqq M(i)$ and $M(k) \subseteq \bigcup_{j \neq i} \alpha_{j}$ imply that $\varphi \uparrow \alpha_{i}$ is a $1-1$ map from $\alpha_{i}$ into $\mathrm{U}_{j \neq i} \alpha_{j}$ and thus $\alpha_{i} \prec_{e} \bigcup_{j \neq i} \alpha_{j}$. But our construction in Theorem 1 ensured $\alpha_{i} \aleph_{e} \cup_{j \neq i} \alpha_{i}$. Thus $M(i) \Varangle_{e} M(k)$ and hence $M(i) \nless_{c} M(k)$ and $M(i) \nless_{i} M(k)$. Thus $M$ is an order preserving map as claimed.

COROLLARY 2.1. Let $\beta$ be any recursively enumerable set which is not recursive and let $P$ be any countable partial ordering. Then there is a collection of co-r.e. bi-dense subsets of $Q$ with property $\mathscr{P}$, each Turing equivalent to $\beta$, such that under $\prec_{c}, \prec_{i}$, or $\prec_{e}$, this collection is order isomorphic to $P$.

Proof. It is a well known result of Mostowski [7] that there is an $\aleph_{0}$-universal recursive partial ordering on $N$. Thus assume that $\left\langle N, \leqq^{*}\right\rangle$ is an $\boldsymbol{\aleph}_{0}$-universal recursive partial ordering on $N$ and let $P=\left\langle\mathscr{C}, \leqq^{* *}\right\rangle$ be any countable partial ordering. If $f: \mathscr{C} \rightarrow N$ be an order preserving map from $P$ to $\left\langle N\right.$, $\left.{ }^{*}\right\rangle$, then $M \circ f$ is an order preserving map from $P$ to $\{M(i) \mid i \in N\}$ under either $\prec_{i},<_{c}$, or $\prec_{e}$. Thus $\{M(i) \mid i \in N\}$ is a collection which satisfies the properties required by the corollary.

COROLlary 2.2. Let $a$ be any nonzero r.e. degree. Then $\left\langle\bar{B}(\boldsymbol{a}, Q), \leqq_{\mathrm{o}}\right\rangle,\left\langle\Lambda_{z}, \leqq_{i}\right\rangle$, and $\left\langle\Lambda_{z}, \leqq_{e}\right\rangle$ are all $\boldsymbol{\aleph}_{0}$-universal partial orderings.

Proof. $\left\langle N, \leqq^{*}\right\rangle$ be as in the proof of Corollary 2.1. Since $i \neq j$ implies either $i \not{ }^{*} j$ or $j \not{ }^{*} i$, it follows that either $M(i) \aleph_{e} M(j)$ or 
$M(j) \prec_{c} M(i)$. Thus $i \neq j$ implies $M(i)$ and $M(j)$ are in distinct equivalence classes mod $\sim_{e}$ and that the recursive equivalence types $\langle M(i)\rangle$ and $\langle M(j)\rangle$ are distinct. Also, since each $M(i)$ has property $\mathscr{P}$, each $M(i)$ is isolated and thus $M(i) \in \Lambda_{z}$.

3. Differences between the partial orderings. First we briefly discuss the differences between $<_{i},<_{c}$, and $<_{e}$ on the co-r.e. subsets of $Q$. We noted earlier that $\forall \alpha, \beta \cong Q\left(\alpha<_{i} \beta \rightarrow \alpha \succ_{c} \beta \rightarrow \alpha \prec_{e} \beta\right)$. We show that none of the reverse implications hold. Let $\tilde{N}=\{\tilde{0}, \widetilde{1}, \widetilde{2}, \cdots\}$ denote the natural numbers as they sit inside of $Q$. Since $\tilde{N}$ is a recursive subset of $Q$, there is a $1-1$ recursive function from $Q$ onto $\widetilde{N}$. Thus $Q \nprec_{e} \widetilde{N}$ but it is clearly the case that $Q \aleph_{c} \tilde{N}$. Next consider the recursive sets $\widetilde{E}=\{\tilde{0}, \widetilde{2}, \widetilde{4}, \cdots\}$ and $\widetilde{D}=\{\tilde{1}, \widetilde{3}, \widetilde{5}, \cdots\}$. Clearly $\widetilde{E} \prec_{c} \widetilde{D}$ but $\widetilde{E} \prec_{i} \widetilde{D}$ since $\widetilde{E} \nsubseteq \widetilde{D}$.

Finally, we give an example to show that $\leqq_{i}$ and $\leqq_{e}$ do not agree on $\Lambda_{z}$. We start with a few definitions. A set $\alpha \subseteq N$ is cohesive $(r$-cohesive) if $\alpha$ is infinite and there is no r.e. (recursive) set $W$ such that $W \cap \alpha$ and $\bar{W} \cap \alpha$ are both infinite. (Note: it follows immediately that if $\alpha$ is cohesive or $r$-cohesive, then $\alpha$ is isolated.) A r.e. set $\beta$ is maximal ( $r$-maximal) if $\bar{\beta}$ is cohesive ( $r$-cohesive). Given r.e. sets $B \subseteq A$ we say $B$ is a major subset of $A$ if $A-B$ is infinite and for any r.e. set $W$ such that $W \cup A=N, N-(W \cup B)$ is finite. Lachlan proves in [6] that every nonrecursive r.e. set has a major subset and that a major subset of a maximal set is an $r$-maximal set. So let $A$ be a maximal set and $B$ be a major subset of $A$. Let $\alpha=\bar{A}$ and $\beta=\bar{B}$. Thus $\alpha$ is cohesive and $\beta$ is $r$-cohesive so that $\langle\alpha\rangle,\langle\beta\rangle \in \Lambda_{z}$. Also $\alpha \subseteq \beta$ so the identity map shows that $\alpha<_{e} \beta$ and hence $\langle\alpha\rangle \leqq_{e}\langle\beta\rangle$. We shall show that $\langle\alpha\rangle \varliminf_{i}\langle\beta\rangle$. Suppose $\langle\alpha\rangle \leqq_{i}\langle\beta\rangle$. Then there are sets $\alpha^{\prime} \in\langle\alpha\rangle$ and $\beta^{\prime} \in\langle\beta\rangle$ such that $\alpha^{\prime} \prec_{i} \beta^{\prime}$. Thus $\alpha^{\prime} \subseteq \beta^{\prime}$ and there are r.e. sets $W_{1}$ and $W_{2}$ such that $W_{1} \cap \beta^{\prime}=\alpha^{\prime}$ and $W_{2} \cap \beta^{\prime}=\beta^{\prime}-\alpha^{\prime}$. Also since $\alpha^{\prime} \in\langle\alpha\rangle$ and $\beta^{\prime} \in\langle\beta\rangle$, there are $1-1$ partial recursive functions $q$ and $p$ such that $\alpha^{\prime} \leqq \delta q$ and $q \uparrow \alpha^{\prime}$ is a $1-1$ map from $\alpha^{\prime}$ onto $\alpha$ and $\beta^{\prime} \subseteq \delta p$ and $p\left\lceil\beta^{\prime}\right.$ is a 1-1 map from $\beta^{\prime}$ onto $\beta$. It must be the case that $\beta^{\prime}-\alpha^{\prime}$ is infinite. For suppose $\beta^{\prime}-\alpha^{\prime}$ is finite. If $\alpha^{\prime \prime}=p\left(\alpha^{\prime}\right)$, then $\beta-\alpha^{\prime \prime}$ is finite and hence $A \cap \alpha^{\prime \prime}$ and $\bar{A} \cap \alpha^{\prime \prime}$ are infinite since $A \cap \beta$ and $\bar{A} \cap \beta$ are infinite. Now $q \circ p^{-1} \uparrow \alpha^{\prime \prime}$ is a $1-1$ map from $\alpha^{\prime \prime}$ onto $\alpha$. Let $U$ be the r.e. set $A \cap \delta q \circ p^{-1}$. Then $q \circ p^{-1}(U)$ is a r.e. set such that $q \circ p^{-1}(U) \cap \alpha \supseteqq q \circ p^{-1}\left(U \cap \alpha^{\prime \prime}\right)$ and $\overline{q \circ p^{-1}(U)} \cap \alpha \supseteqq q \circ p^{-1}(\bar{U} \cap \alpha)$. Thus

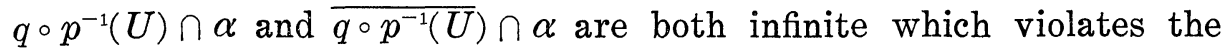
fact that $\alpha$ is cohesive. Next, consider the r.e. sets $U_{1}=W_{1} \cap \delta p$ and $U_{2}=W_{2} \cap \delta p$. Then $p\left(U_{1}\right)$ and $p\left(U_{2}\right)$ are r.e. sets and $p\left(U_{1}\right) \cap \beta \supseteqq$ $p\left(U_{1} \cap \beta^{\prime}\right)=p\left(\alpha^{\prime}\right)$ and $p\left(U_{2}\right) \cap \beta \supseteqq p\left(U_{2} \cap \beta^{\prime}\right)=p\left(\beta^{\prime}-\alpha^{\prime}\right)$. Thus 
$p\left(U_{1}\right) \cap \beta$ and $p\left(U_{2}\right) \cap \beta$ are both infinite. Now let $V_{1}=B \cup p\left(U_{1}\right)$ and $V_{2}=B \cup p\left(U_{2}\right)$. Note that $U_{1} \cup U_{2} \supseteqq \delta p \supseteqq \beta^{\prime}$ and hence $p\left(U_{1}\right) \cup$ $p\left(U_{2}\right) \supseteqq \beta=N-B$ which implies $V_{1} \cup V_{2}=N$. From the enumerations of $V_{1}$ and $V_{2}$, we can construct recursive sets $R_{1}$ and $R_{2}$ as follows. We put $x$ in $R_{1}$ if $x$ is enumerated in $V_{1}$ before it is enumerated in $V_{2}$ and put $x$ in $R_{2}$ otherwise. Then $\bar{R}_{1}=R_{2}$ and $R_{1} \cap \beta=V_{1} \cap \beta=$ $p\left(U_{1}\right) \cap \beta$ and $R_{2} \cap \beta=V_{2} \cap \beta=p\left(U_{2}\right) \cap \beta$. Thus $R_{1}$ violates the fact that $\beta$ is $r$-cohesive. Thus $\langle\alpha\rangle \$_{2}\langle\beta\rangle$ and we have proved the following.

THEOREM 3 . $\varliminf_{i}$ and $\leqq_{e}$ do not agree on $\Lambda_{z}$.

We wish to thank A. B. Manaster for introducing us to this problem and for helpful conversations.

\section{REFERENCES}

1. J.C. E. Dekker and J. Myhill, Recursive equivalence types, (monograph), University of California publications in mathematics, n.s. vol. 3, 1960.

2. E. Ellentuck, Universal cosimple isols, Pacific J. Math., 42 (1972), 629-638.

3. L. S. Hay, The co-simple isols, Ann. of Math., 83 (1966), 231-256.

4. - Elementary differences between the isols and the co-simple isols, Trans. Amer. Math. Soc., 127 (1967), 427-441.

5. L. S. Hay, A. B. Manaster and J. G. Rosenstein, Concerning partial recursive similarity transformations of linearly ordered sets, Pacific J. Math., 71 (1977), 57-70. 6. A. H. Lachlan, On the lattice of recursively enumerable sets, Trans. Amer. Math. Soc., 130 (1968), 1-27.

7. A. Mostowski, Über gewise universelle relationen, Ann. Soc. Polon. Math., 17 (1938), 117-118.

8. G. E. Sacks, Degrees of Unsolvability, (monograph), Annals of Mathematics Studies No. 55, Princeton Univ. Press, Princeton, N. J., 1963.

Received February 2, 1977, and in revised form August 19, 1977.

University of California, San Diego

LA JOLLA, CA 92093 


\section{PACIFIC JOURNAL OF MATHEMATICS}

\section{EDITORS}

RICHARD ARENS (Managing Editor)

University of California

Los Angeles, California 90024

C. W. CURTIS

University of Oregon

Eugene, OR 97403

C. C. MOORE

University of California

Berkeley, CA 94720

\section{J. DUGUNDJI}

Department of Mathematics University of Southern California Los Angeles, California 90007

R. Finn aNd J. Milgram Stanford University Stanford, California 94305

\section{ASSOCIATE EDITORS}

E. F. BeCKenbaCH

B. H. NeumanN

F. WOLF

K. YosHIDA

\section{SUPPORTING INSTITUTIONS}

UNIVERSITY OF BRITISH COLUMBIA CALIFORNIA INSTITUTE OF TECHNOLOGY UNIVERSITY OF CALIFORNIA MONTANA STATE UNIVERSITY UNIVERSITY OF NEVADA, RENO NEW MEXICO STATE UNIVERSITY OREGON STATE UNIVERSITY UNIVERSITY OF OREGON
UNIVERSITY OF SOUTHERN CALIFORNIA STANFORD UNIVERSITY UNIVERSITY OF HAWAII UNIVERSITY OF TOKYO UNIVERSITY OF UTAH WASHINGTON STATE UNIVERSITY UNIVERSITY OF WASHINGTON 


\section{Pacific Journal of Mathematics}

\section{Vol. 76, No. $1 \quad$ November, 1978}

Ata Nuri Al-Hussaini, Potential operators and equimeasurability ......... 1

Tim Anderson and Erwin Kleinfeld, Semisimple nil algebras of type $\delta . \ldots .99$

Stephen LaVern Campbell, Linear operators for which $T^{*} T$ and $T+T^{*}$

commute. III ......................................

Robert Jay Daverman, Special approximations to embeddings of codimension one spheres...............................

Donald M. Davis, Connective coverings of $\mathrm{BO}$ and immersions of projective

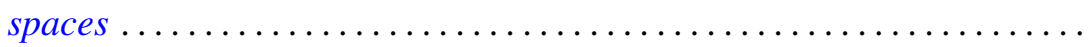

V. L. (Vagn Lundsgaard) Hansen, The homotopy type of the space of maps of

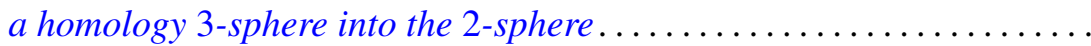

James Victor Herod, A product integral representation for the generalized

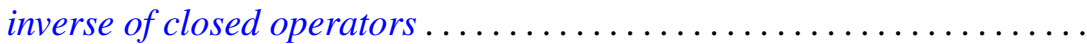

A. A. Iskander, Definability in the lattice of ring varieties ..............

Russell Allan Johnson, Existence of a strong lifting commuting with a

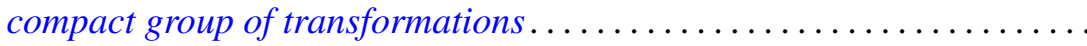

Heikki J. K. Junnila, Neighbornets...................... 83

Klaus Kalb, On the expansion in joint generalized eigenvectors . ......... 109

F. J. Martinelli, Construction of generalized normal numbers . . . . . . . . . 117

Edward O'Neill, On Massey products ....................... 123

Vern Ival Paulsen, Continuous canonical forms for matrices under unitary

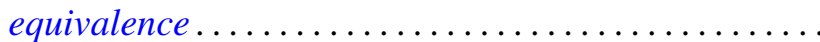

Justin Peters and Terje Sund, Automorphisms of locally compact groups . . . 143

Duane Randall, Tangent frame fields on spin manifolds . . . .

Jeffrey Brian Remmel, Realizing partial orderings by classes of co-simple sets . . . .

J. Hyam Rubinstein, One-sided Heegaard splittings of 3-manifolds ...

Donald Charles Rung, Meier type theorems for general boundary approach

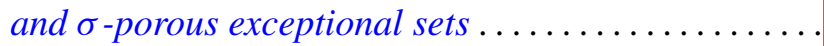

Ryōtarō Satō, Positive operators and the ergodic theorem

Ira H. Shavel, A class of algebraic surfaces of general type constructed from

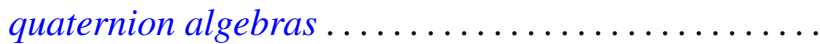

Patrick F. Smith, Decomposing modules into projectives and injectives ....

Sergio Eduardo Zarantonello, The sheaf of outer functions in the polydisc... 